\title{
Cryptogenic Gelastic Epilepsy Originating from the Right Temporal Lobe
}

\author{
Yaohui Chai Bola Adamolekun \\ Department of Neurology, University of Tennessee Health Science Center, Memphis, Tenn., USA
}

\section{Key Words \\ Gelastic epilepsy · Diagnosis · Video-EEG $\cdot$ Treatment}

\begin{abstract}
Objective: To present a case of intractable cryptogenic gelastic epilepsy with ictal video-EEG to localize the seizure focus. Clinical Presentation and Intervention: A 39-yearold female presented with a 2-year history of intractable gelastic epilepsy characterized by recurrent episodes of stereotyped pathological laughter, transient unresponsiveness, automatisms and brief postictal confusion. The patient failed to respond to multiple antiepileptic drugs. Several interictal EEGs did not show any abnormalities. Magnetic resonance imaging of the head with seizure protocol and diffusion tensor imaging were normal. Ictal video-EEG monitoring showed rhythmic, sharp activity emanating from the right temporal lobe. Conclusion: This case suggests that the right temporal lobe may be actively involved in the epileptogenic network generating gelastic epilepsy. Video-EEG monitoring should be considered for patients with gelastic epilepsies to better clarify the nature of events, localize the seizure focus and correlate EEG changes with the clinical manifestations.

Copyright $\odot 2010$ S. Karger AG, Basel
\end{abstract}

\section{Introduction}

Gelastic epilepsy is characterized by stereotyped pathologic laughter, absence of external precipitants, concomitant epileptic manifestations, ictal epileptiform discharges and absence of other neuropathology that could explain pathologic laughter [1]. Gelastic epilepsy is very rare. It is most commonly associated with hypothalamic hamartomas, but has also been reported to occur with lesions and tumors in the temporal and frontal lobes. However, it may also be cryptogenic, of unknown origin and without evidence of structural lesions of the central nervous system [2-4]. In this paper, we report a case of intractable, cryptogenic gelastic epilepsy with ictal video-EEG localization of the seizure focus to the right temporal lobe.

\section{Case Report}

A 39-year-old right-handed female was admitted to the comprehensive seizure monitoring unit for video-EEG monitoring as part of a presurgical evaluation for intractable seizures. Prior to admission, the patient experienced a 2-year history of seizures starting with laughing and giggling, and accompanied by altered awareness, transient staring, automatisms including lip smacking and hand picking. She seemed to be joyful and happy during the episode and had brief postictal confusion afterwards. The patient had failed to respond to several antiepileptic drugs including phenytoin, trileptal, levetiracetam and zonisamide. At presentation, she was having approximately 3 seizures per month while on polytherapy with therapeutic doses of lamotrigine, topiramate and pregabalin. Past medical, family and social histories were unremarkable. Physical and neurological examinations were normal.

\section{KARGER \\ Fax +4161306 1234 \\ E-Mail karger@karger.ch}

www.karger.com
C 2010 S. Karger AG, Basel

$1011-7571 / 10 / 0192-0153 \$ 26.00 / 0$

Accessible online at:

www.karger.com/mpp
Dr. Bola Adamolekun

Department of Neurology, 415 Link Building

University of Tennessee Health Science Center

855 Monroe Avenue, Memphis, TN 38163 (USA)

Tel. +1 901448 4916, Fax +1 901448 7440, E-Mail badamole@ utmem.edu 


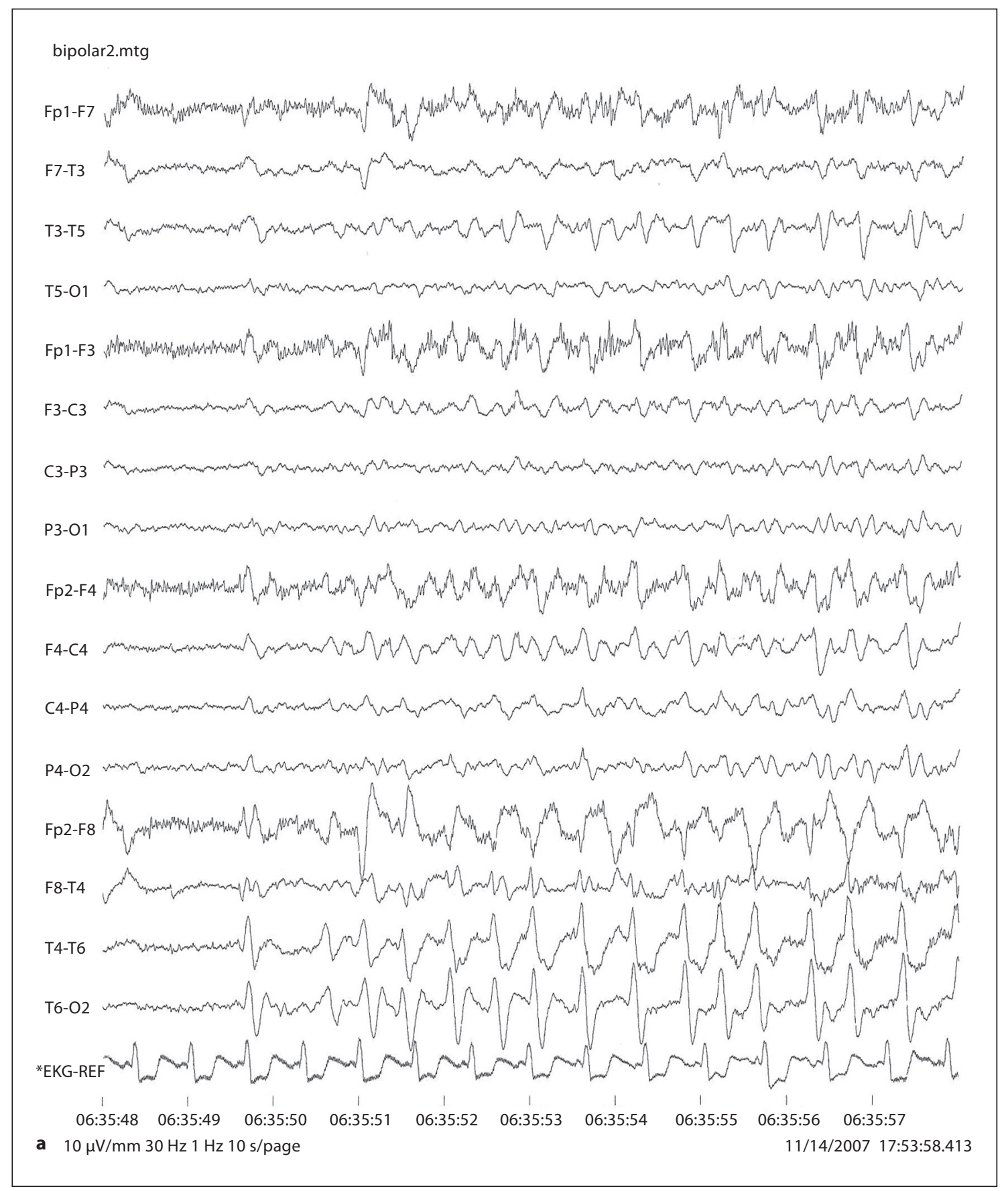

Fig. 1. Ictal video-monitoring EEG of a gelastic seizure by using bipolar montage, showing sharp and slow waves emanating from the right temporal lobe (a) with subsequent bilateral spread (b).

Several interictal EEGs did not show any epileptiform abnormalities. Cerebral magnetic resonance imaging (MRI) with seizure protocol was performed in a 1.5 -tesla scanner. Sagittal $\mathrm{T}_{1^{-}}$ weighted axial diffusion, $\mathrm{T}_{2}$-weighted imaging and fluid-attenuated inversion recovery as well as coronal fluid-attenuated inversion recovery, $\mathrm{T}_{2}$-weighted fast spin echo and 3-dimension- al spoiled gradient recalled acquisition in steady state (3D-SPGR) imaging were performed. $\mathrm{T}_{1}$-weighted SPGR images were obtained at $1.5-\mathrm{mm}$ slice thickness with no intervening gap. After intravenous administration of a standard dose of gadoliniumDTPA contrast agent, axial $\mathrm{T}_{1}$-weighted spine echo images were obtained through the brain. MRI did not detect any structural 


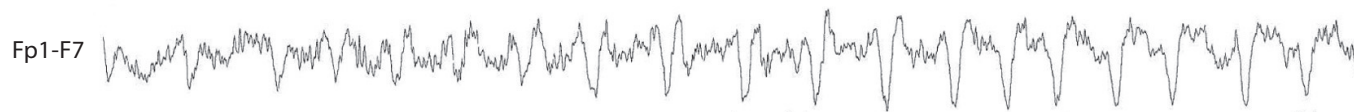

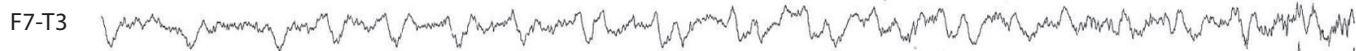

T3-T5 JMand

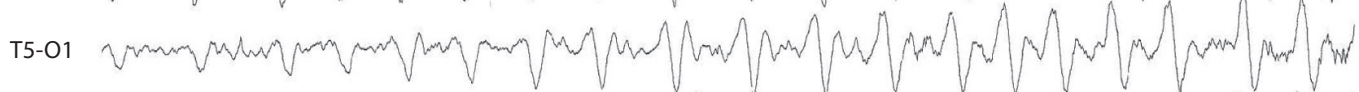

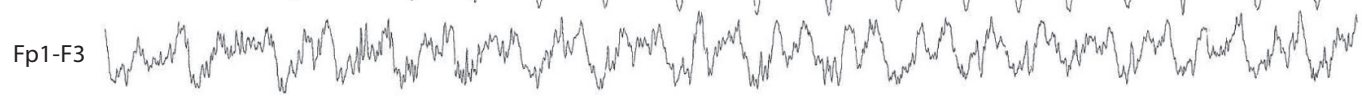

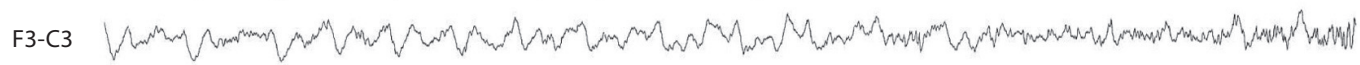

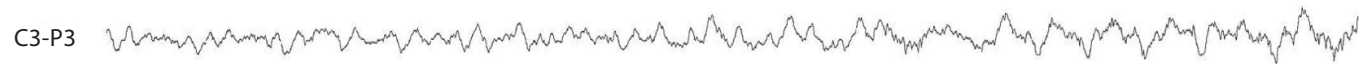

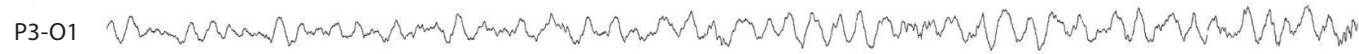

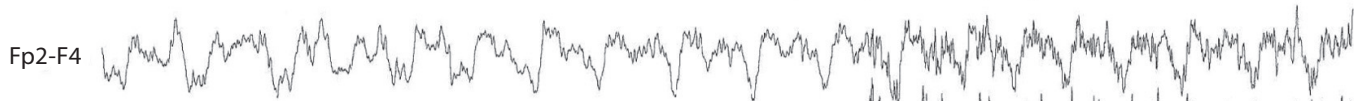

F4-C4 Aw

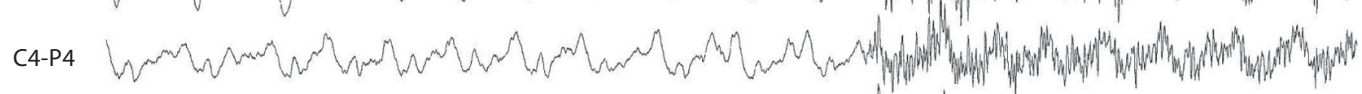

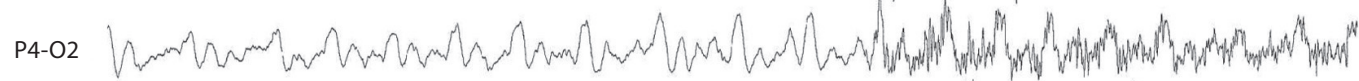

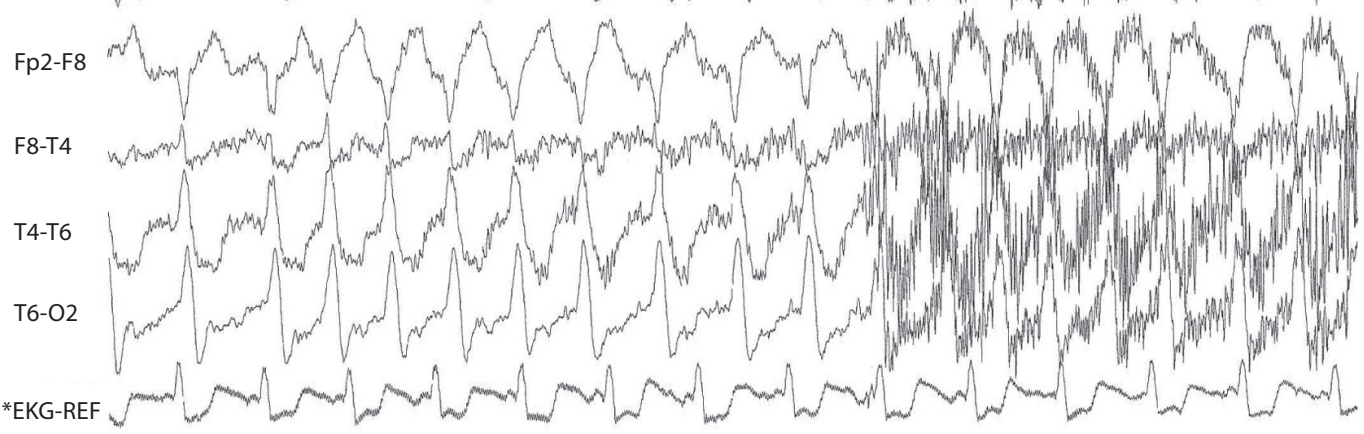

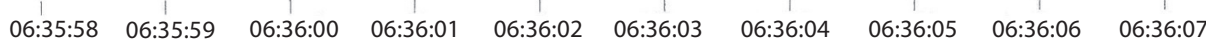

b $10 \mu \mathrm{V} / \mathrm{mm} 30 \mathrm{~Hz} 1 \mathrm{~Hz} 10 \mathrm{~s} /$ page

abnormalities in the brain. Additional diffuse tensor imaging (DTI) was also performed. DTI showed there was no abnormality in fractional anisotropy and mean diffusivity throughout the brain. Position emission tomography (PET) and single photon emission computed tomography imaging studies were not done because of insurance issues. The patient's antiepileptic medica- tions were tapered off prior to admission for seizure monitoring. During 4 days of video-EEG monitoring, a total of 5 episodes of gelastic epilepsy were observed. The clinical semiology of the seizures was as described above. The ictal EEG correlates of these events were rhythmic, sharp waves emanating from the right temporal lobe (fig. 1a, 2a) and then spreading to become bilat- 


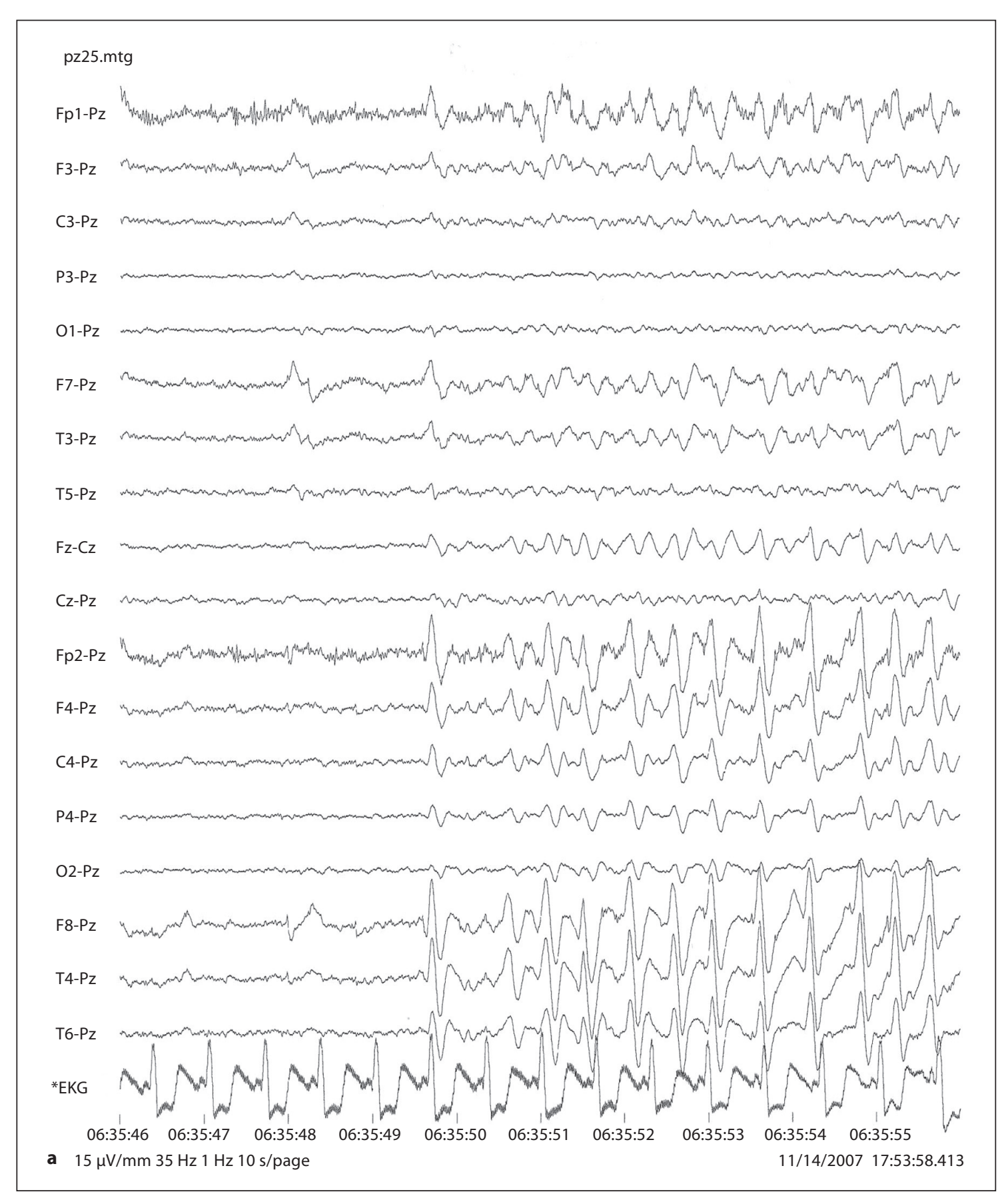

Fig. 2. Ictal video-monitoring EEG of a gelastic seizure by using referential montage, showing sharp and slow waves emanating from the right temporal lobe (a) with subsequent bilateral spread (b).

eral (fig. 1b, 2b). The patient was offered the option of subdural electrode strip placement for intracranial seizure monitoring. However, she worried about the invasiveness of this procedure and preferred the medical treatment at this time even though it had been proved to be ineffective.

\section{Discussion}

It has been suggested that normal laughter is the result of an interaction of several different brain structures: the frontal and temporal neocortex; the temporobasal cortex; 


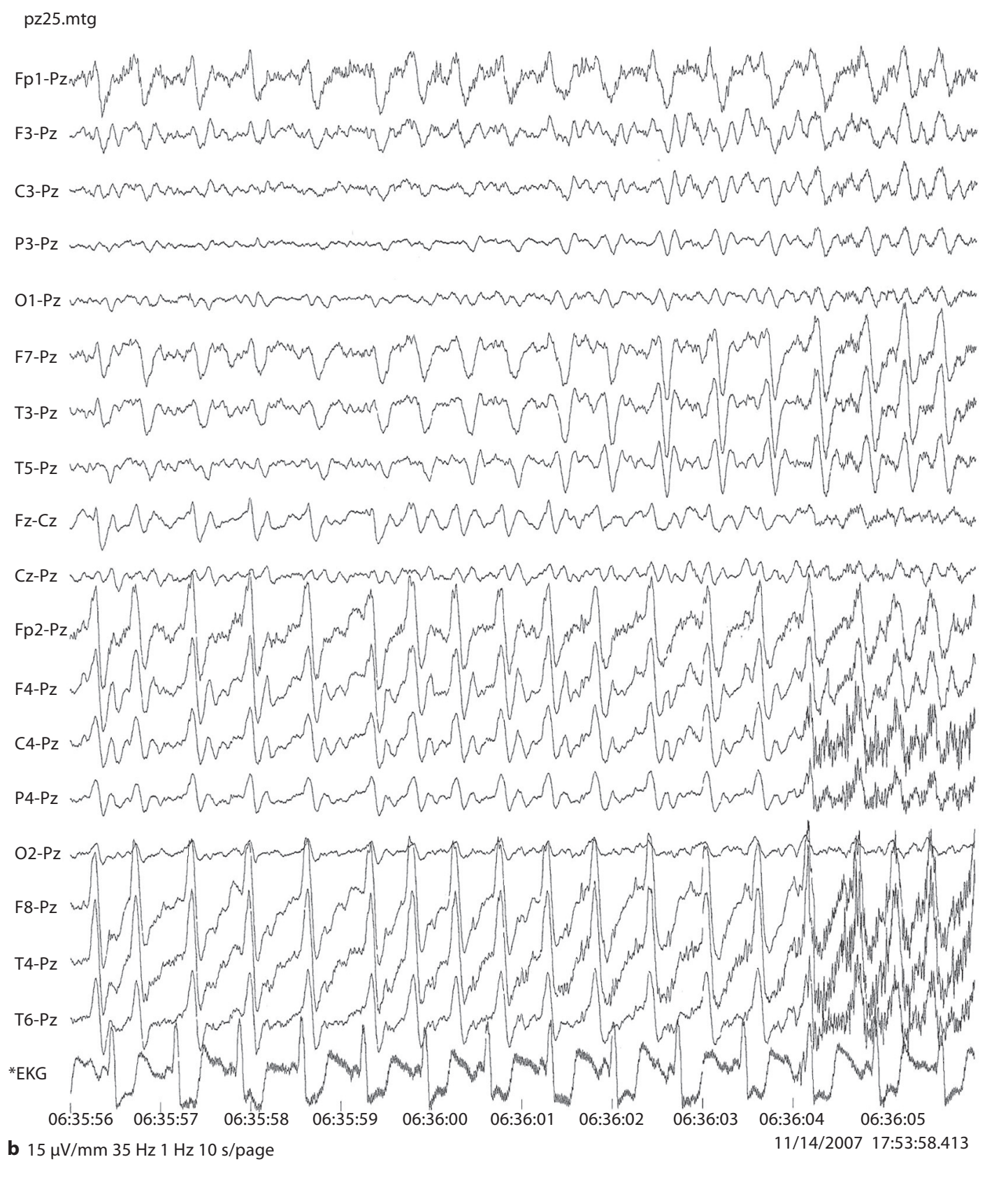

the visual, olfactory, and auditory associative areas; the limbic system with the cingulate gyrus; and the brainstem [5, 6]. However, it has been shown that cryptogenic gelastic seizures may originate from either side of the frontal areas and then spread to the adjacent frontotem- poral region $[2,4]$ or may originate from unspecifed sides of the temporal areas [3]. In our case, ictal video-EEG showed cryptogenic gelastic seizures clearly starting in the right temporal lobe then spreading to the bilateral hemispheres. This suggests that the right temporal lobe 
may be actively involved in the epileptogenic network generating gelastic seizures.

Gelastic epilepsy could be misdiagnosed as gastroesophageal reflux disease or psychiatric diseases $[7,8]$. In our case, several ambulatory interictal EEGs did not show any epileptiform abnormality. However, ictal video-EEG monitoring confirmed the epileptic etiology of the patient's pathologic laughter. Thus, continuous video-EEG monitoring is an essential investigation for the characterization of gelastic seizures, for the localization of the seizure focus and for the correlation of EEG changes with the clinical manifestations. The distinction between epileptic and nonepileptic laughter can be facilitated by the use of video-EEG monitoring.

The antiepileptic drugs used to treat partial seizures may also be used in treating gelastic epilepsy. However, none of these medications are likely to lead to complete seizure control [9]. As in this case, multiple antiepileptic drugs may be needed for seizure control. Currently, early individualized surgical management remains the mainstay for gelastic seizures originating from a hypothalamic hamartoma [9]. Direct resection, interstitial radiosurgery or gamma knife radiosurgery for hamartoma have gained fair success in stopping or reducing the frequency of this gelastic epilepsy $[9,10]$. However, there are limited studies on the therapeutic options available for intractable cryptogenic gelastic epilepsy. Moon et al. [4] reported that patients with cryptogenic gelastic epilepsy had been seizure free after resection of the medial frontal area, including the epileptogenic focus. Based on this report, it could be inferred that localization of the focus of cryptogenic gelastic epilepsy by intracranial EEG monitoring followed by regional resection will be the best option for the control of these intractable seizures.

A seizure protocol MRI of the head with DTI sequences is an essential investigation to exclude structural lesions before a diagnosis of cryptogenic gelastic epilepsy can be made. DTI has been shown to be more sensitive than conventional MRI in the detection of subtle cortical malformations and microstructural abnormalities in epileptic foci [11]. Hypermetabolic regions have been found within the area of the hypothalamic harmatoma on PET scanning in a patient with gelastic epilepsy [12]. A PET scan may be useful in confirming the absence of focal lesions in patients with cryptogenic epilepsy.

\section{Conclusion}

This report described a case of intractable cryptogenic gelastic epilepsy emanating from the right temporal lobe, thereby indicating that the right temporal lobe may be actively involved in the epileptogenic network generating gelastic epilepsy. Video-EEG monitoring should be considered for gelastic epilepsy to better clarify the nature of events, localize the seizure focus and correlate EEG changes with the clinical manifestations.

\section{Acknowledgement}

The authors thank Dr. Michael Jacewicz for critical reading of the manuscript.

\section{References}

1 Freeman JL, Eeg-Olofsson O: Gelastic seizures; in Engel J, Pedley TA (eds): Epilepsy: A Comprehensive Textbook, ed 2. Philadelphia, Lippincott Williams \& Wilkins, 2007, pp 619-623.

-2 Garcia A, Gutiérrez MA, Barrasa J, Herranz JL: Cryptogenic gelastic epilepsy of frontal lobe origin: a paediatric case report. Seizure 2000;9:297-300.

- 3 Striano S, Meo R, Bilo L, Cirillo S, Nocerino C, Ruosi P, Striano P, Estraneo A: Gelastic epilepsy: symptomatic and cryptogenic cases. Epilepsia 1999;40:294-302.

4 Moon SY, Seo DW, Kim HY, Hong SC, Hong SB: Cryptogenic gelastic seizures from medial frontal area: 2 cases. J Korean Neurol Assoc 2002;20:716-720.
5 Lopes da Silva FH, Witter MP, Boeijinga PH, Lohman AH: Anatomic organization and physiology of the limbic cortex. Physiol Rev 1990;70:453-511.

-6 Arroyo S, Lesser RP, Gordan B, Arroyo S, Lesser RP, Gordon B, Uematsu S, Hart J, Schwerdt P, Andreasson K, Fisher RS: Mirth, laughter and gelastic seizures. Brain 1993; 116:757-780.

7 Sweetman LL: Gelastic seizures misdiagnosed as gastroesophageal reflux disease. Clin Pediatr 2007;46:325-328.

-8 Glassman JN, Dryer D, McCartney JR: Complex partial status epilepticus presenting as gelastic seizures: a case report. Gen Hosp Psychiatry 1986;8:61-64.

-9 Deopujari CE, Suhas U: Surgical considerations in the management of gelastic seizures. J Pediatr Neurosci 2008;3:88-93.
10 Schulze-Bonhage A, Homberg V, Trippel M, Keimer R, Elger CE, Warnke PC, Ostertag C: Interstitial radiosurgery in the treatment of gelastic epilepsy due to hypothalamic hamartomas. Neurology 2004;62:644-647.

11 Dumas de la Roque A, Oppenheim C, Chassoux F, Rodrigo S, Beuvon F, Daumas Duport C, Devaux B, Meder JF: Diffusion tensor imaging of partial intractable epilepsy. Eur Radiol 2005;15:279-285.

12 Shahar E, Goldsher D, Genizi J, Ravid S, Keidar Z: Intractable gelastic seizures during infancy: ictal positron emission tomography (PET) demonstrating epileptiform activity within the hypothalamic hamartoma. J Child Neurol 2008;23:235-239. 